\title{
First-principles study of the structural, electronic, vibrational, and elastic properties of orthorhombic NiSi
}

\author{
D. Connétable ${ }^{1, *}$ and $\mathrm{O}$. Thomas ${ }^{2,3}$ \\ ${ }^{1}$ CIRIMAT CNRS/INPT/UPS (UMR 5085), ENSIACET, 118 Route de Narbonne, F-31077 Toulouse Cedex 4, France \\ ${ }^{2}$ Aix-Marseille Université, IM2NP \\ ${ }^{3}$ Faculté des Sciences et Techniques, CNRS, IM2NP (UMR 6242), Campus de St Jérome, F-13397 Marseille Cedex, France
}

(Received 14 April 2008; revised manuscript received 28 November 2008; published 3 March 2009)

\begin{abstract}
We present a study of the structural, electronic, vibrational, and elastic properties of the orthorhombic NiSi structure by means of the density-functional theory and the density-functional perturbative theory, with the Perdew-Burke-Ernzerhof generalized gradient approximation of the exchange-correlation functional, within its spin-polarized version. The optimized lattice parameters, the formation energy, and vibrational properties are found in agreement with experimental data. We show that NiSi is not ferromagnetic, with a low density of states at the Fermi level. Elastic constants have been calculated by means of three different approaches for comparison. In the first two, the calculated energy $E$ is fitted as a function of the deformation, atomic positions are either relaxed or not relaxed during the simulations. Atomic relaxations are shown to modify significantly elastic constants. In the third approach we have related acoustic velocities to elastic constants. NiSi is shown to be highly anisotropic. In particular the linear bulk modulus along $b$ axis is much larger than along other axes. Polycrystalline elastic properties and Debye temperature have been also evaluated for a complete description of elastic properties.
\end{abstract}

DOI: 10.1103/PhysRevB.79.094101

PACS number(s): 62.20.-x, 61.66.Dk, 71.15.Mb

\section{INTRODUCTION}

NiSi has attracted a lot of attention recently because it is used as a contact material in advanced electron devices. ${ }^{1}$ Indeed it exhibits a low resistivity of about $10 \mu \Omega / \mathrm{cm}$ at room temperature. ${ }^{2} \mathrm{NiSi}$ crystallizes in the orthorhombic, MnP-type structure (noted $o$-NiSi) and its few known physical properties appear to be highly anisotropic. This is the case of thermal expansion where one of the three principal components (along the $b$ axis) of the thermal expansion tensor is negative. ${ }^{3}$ The elastic constants are not known and one may wonder whether NiSi is a highly elastically anisotropic material. This may bear important consequences on, on one hand, the mechanical behavior of NiSi polycrystalline thin films, and on the other hand, the stresses that arise in silicon as a consequence of silicide formation. ${ }^{4}$ These stresses have a strong influence on the mobility of charge carriers in silicon. ${ }^{5}$ This study is aimed at calculating the elastic constants of NiSi from first principles. The first part is devoted to the methodology of elastic constants calculation including both numerical methods and basics of elasticity. The obtained results are then described and discussed. Their consequences on the mechanics of NiSi layers are highlighted.

\section{METHODOLOGY}

\section{A. Computational details}

The ground states of the undeformed $o$-NiSi and reference systems (Ni-fcc and silicon diamond phases) have been optimized by means of the computational implementation of the density-functional theory (DFT) VASP (Ref. 6) and QUANTUM-ESPRESSO (Ref. 7) packages for comparison. We have used projected-augmented wave (PAW) pseudopotentials $^{8}$ with VASP and ultrasoft (US) pseudopotentials ${ }^{9}$ according to a modified Rappe-Rabe-
Kaxiras-Joannopoulos scheme following the method of Ref. 10 for the ESPRESSO package (noted in the following PAW and US simulations, respectively). The Perdew-BurkeErnzerhof (Ref. 11) generalized gradient approximation of the exchange-correlation energy has been used within its spin-polarized version.

The Brillouin zones have been sampled by a $15 \times 15$ $\times 15$ Monkhorst and Pack grid ${ }^{12}$ for nickel and silicon and $8 \times 8 \times 8$ for NiSi for structure optimizations. The $500 \mathrm{eV}$ and 40 Ryd energy cutoff for VASP and ESPRESSO packages, respectively, have been employed. To optimize lattice parameters, the conjugate gradient method have been employed with VASP, while the equilibrated structure for ESPRESSO simulations was calculated using the first-principles implementation of a variable cell-shape damped molecular dynamics. ${ }^{13}$

For the calculation of the electronic density of states and the formation energy, we have adopted a finest tetrahedron $30 \times 30 \times 30(15 \times 15 \times 15$ for NiSi $)$ grid with a higher energy cutoff (600 eV and 50 Ryd).

\section{B. Elastic constants}

For small strains $(\epsilon)$, Hooke's law applies and the elastic energy $E$ can be approximated by a quadratic function of the strain components:

$$
E \propto V_{o} \sum_{i, j=1}^{6} \frac{1}{2} C_{i j} e_{i} e_{j},
$$

where $C_{i j}$ are the elastic constants, $V_{o}$ is the equilibrium volume of the unit cell, and $e_{j}$ is the components of the strain tensor $(\epsilon)$ which can be related to the deformation tensor $\mathbf{D}$ by 
TABLE I. Components of the strain tensor, elements not given are zero. The first parts are the components for cubic systems, and the second are the nine relations for orthorhombic systems $\left[u=\left(1-\delta^{2}\right)^{-1 / 3}\right]$.

\begin{tabular}{lcccccc}
\hline \hline \multicolumn{7}{c}{ Cubic systems } \\
\hline$e_{1}$ & $e_{2}$ & $e_{3}$ & $e_{4}$ & $e_{5}$ & $e_{6}$ & $E(\delta)$ \\
\hline$\delta$ & $\delta$ & $\delta$ & 0 & 0 & 0 & $9 B_{o} V_{o} \delta^{2} / 2$ \\
$\delta$ & 0 & 0 & 0 & 0 & 0 & $V_{o} C_{11} \delta^{2} / 2$ \\
0 & 0 & 0 & $2 \delta$ & $2 \delta$ & $2 \delta$ & $6 V_{0} C_{44} \delta^{2}$
\end{tabular}

\begin{tabular}{lcccccc}
\multicolumn{7}{c}{ Orthorhombic systems } \\
$e_{1}$ & $e_{2}$ & $e_{3}$ & $e_{4}$ & $e_{5}$ & $e_{6}$ & $E(\delta)$ \\
\hline$\delta$ & 0 & 0 & 0 & 0 & 0 & $V_{o} C_{11} \delta^{2} / 2$ \\
0 & $\delta$ & 0 & 0 & 0 & 0 & $V_{o} C_{22} \delta^{2} / 2$ \\
0 & 0 & $\delta$ & 0 & 0 & 0 & $V_{o} C_{33} \delta^{2} / 2$ \\
0 & 0 & 0 & $2 \delta$ & 0 & 0 & $2 V_{o} C_{44} \delta^{2}$ \\
0 & 0 & 0 & 0 & $2 \delta$ & 0 & $2 V_{o} C_{55} \delta^{2}$ \\
0 & 0 & 0 & 0 & 0 & $2 \delta$ & $2 V_{o} C_{66} \delta^{2}$ \\
$u(1+\delta)-1$ & $u(1-\delta)-1$ & $u-1$ & 0 & 0 & 0 & $V_{o}\left(C_{11}+C_{22}-2 C_{12}\right) \delta^{2} / 2$ \\
$u(1+\delta)-1$ & $u-1$ & $u(1-\delta)-1$ & 0 & 0 & 0 & $V_{o}\left(C_{11}+C_{33}-2 C_{13}\right) \delta^{2} / 2$ \\
$u-1$ & $u(1+\delta)-1$ & $u(1-\delta)-1$ & 0 & 0 & 0 & $V_{o}\left(C_{22}+C_{33}-2 C_{23}\right) \delta^{2} / 2$ \\
\hline \hline
\end{tabular}

$$
\boldsymbol{\epsilon}=\mathbf{D}-\mathbf{I}=\frac{1}{2}\left(\begin{array}{ccc}
2 e_{1} & e_{6} & e_{5} \\
e_{6} & 2 e_{2} & e_{4} \\
e_{5} & e_{4} & 2 e_{3}
\end{array}\right)
$$

in conventional elastic theory notation (I represents the identity matrix).

\section{Cubic crystals}

For cubic crystals, there are three independent elastic constants, namely, $C_{11}, C_{12}$, and $C_{44}$. Furthermore, the bulk modulus $B_{0}$ and tetragonal shear constant $C^{\prime}$ are related to $C_{11}$ and $C_{12}$, i.e., $B_{0}=\left(C_{11}+2 C_{12}\right) / 3$ (in the Voigt notation) and $C^{\prime}=\left(C_{11}-C_{12}\right) / 2$, respectively. $C_{44}$ is related to the trigonal shear constant. These constants may be determined by a polynomial fitting of the calculated energy $E$ as a function of the deformation related to the strains presented in Table I.

\section{Orthorhombic crystals}

For orthorhombic crystals, there are nine independent elastic constants, namely, $C_{11}, C_{22}, C_{33}, C_{44}, C_{55}, C_{66}, C_{12}$, $C_{13}$, and $C_{23}$. We need then nine different (strains) relations to determine these.

To evaluate elastic constants in an orthorhombic system, we have used three different techniques. The first two methods use deformations as proposed by Ravindran ${ }^{14}$ (see Table I). We have applied deformations with atomic relaxations or without atomic relaxations. The $15 \times 15 \times 15$ grids with 500 eV (PAW simulations) or 40 Ryd (US) energy cutoffs have been used for these methods.

In the third method, we have calculated vibrational properties by means of the density-functional perturbative theory
(DFPT) (Ref. 15) [ESPRESSO (Ref. 7) simulations] and related acoustic slopes-sound velocities-and elastic constants. We have employed the same method as described by Every ${ }^{16}$ to calculate the diagonal elements of the elastic matrix $\left(C_{i i}\right)$. Along the [100], [010], and [001] directions, we can deduce six independent relations: $v_{l}^{[100]}=\sqrt{C_{11} / \rho}, v_{t 1}^{[100]}$ $=\sqrt{C_{66} / \rho}, v_{t 2}^{[100]}=\sqrt{C_{55} / \rho}, v_{t 2}^{[010]}=\sqrt{C_{44} / \rho}, v_{l}^{[010]}=\sqrt{C_{22} / \rho}$, and $v_{l}^{[001]}=\sqrt{C_{33} / \rho}$, where $v_{l}$ and $v_{t}$ correspond, respectively, to the longitudinal and transverse acoustic sound velocities. However, for the nondiagonal terms $\left(C_{12}, C_{13}\right.$, and $\left.C_{23}\right)$ the relations are more difficult to be calculated due to the nonorthonormality of the vectors of the unit cell.

\section{Crystal structure}

$\mathrm{NiSi}$ has a simple orthorhombic primitive cell belonging to the Pnma, No. 62 (oP8 Pearson symbol) space group. The orthorhombic unit cell contains four nickel and four silicon atoms (see Fig. 1). The optimized crystallographic atomic positions are reported in Table II. Nickel atoms have six first silicon neighbors forming a strongly distorted octahedron, and silicon atoms have six first neighbors (nickel atoms) forming a distorted trigonal prism.

\section{RESULTS AND DISCUSSION}

\section{A. Structural and cohesive properties}

We have used silicon diamond and ferromagnetic $\mathrm{Ni}$ fcc phases as reference states. The lattice parameters, the magnetism, and the bulk modulus have been reported in Table III. The ground states of these systems are found in satisfactory agreement with literature.

For the NiSi orthorhombic system, our optimized structures (at $0 \mathrm{~K}$ ) are in good agreement with experimental lat- 


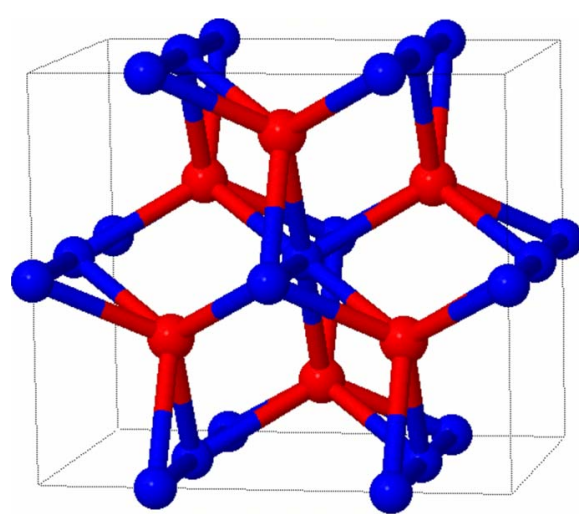

FIG. 1. (Color online) Schematic representation of the NiSi orthorhombic unit cell: in blue: nickel atoms, and in red: silicon atoms.

tice parameters $a_{o}, b_{o}$, and $c_{o}$. Discrepancies of less than $1 \%$ are evidenced in comparison with Wilson's ${ }^{3}$ measurements at $300 \mathrm{~K}$, with a volume of the unit cell which is larger than the experimental value. When comparing with values measured on supported thin films ${ }^{4,19}$ the presence of residual stresses makes the comparison less reliable.

The formation energy has been evaluated to be around $-500 \mathrm{meV} /$ atom. This value is consistent with measured or assessed values $[\simeq-447 \mathrm{meV} /$ atom (Refs. 20 and 21$)]$. One notes that US and PAW simulations give the same lattice parameters and formation energy. The magnetism of $o-\mathrm{NiSi}$ has been investigated too. It is found to be nonferromagnetic, in agreement with experimental findings. ${ }^{2}$

The electronic band structure $(e \mathrm{BS})$ and density of states have been also evaluated (Fig. 2). The $e \mathrm{BS}$ has been plotted along high-symmetry points of the first Brillouin zone represented in Fig. 3. Our results and Boulet simulations ${ }^{17}$ are found in excellent agreement.

It is interesting to note that despite the presence of a cluster of bands in the band structure of $\mathrm{NiSi}$, only two degen-

TABLE II. Crystallographic positions of nickel and silicon atoms in the unit cell. We report our optimized and Boulet (Ref. 17) atomic positions (US and PAW for ESPRESSO and VASP simulations, respectively).

\begin{tabular}{lccc}
\hline \hline \multicolumn{4}{c}{ oP8 } \\
\hline Atom & $x$ & $y$ & $z$ \\
\hline$A_{1}$ & $x$ & 0.25 & $z$ \\
$A_{2}$ & $-x$ & 0.75 & $-z$ \\
$A_{3}$ & $0.5-x$ & 0.75 & $0.5+z$ \\
$A_{4}$ & $0.5+x$ & 0.25 & $0.5-z$ \\
$\mathrm{Ni}^{\mathrm{a}}$ & & $x=0.006 ; z=0.184$ & \\
$\mathrm{Si}^{\mathrm{a}}$ & & $x=0.170 ; z=0.580$ & \\
$\mathrm{Ni}$ (PAW) & & $x=0.008 ; z=0.189$ & \\
$\mathrm{Si}$ (PAW) & & $x=0.179 ; z=0.584$ & \\
$\mathrm{Ni}$ (US) & & $x=0.008 ; z=0.188$ & \\
$\mathrm{Si}$ (US) & & $x=0.179 ; z=0.584$ & \\
\hline \hline
\end{tabular}

${ }^{\mathrm{a}}$ Reference 17. erate bands cross the Fermi level in most of the symmetry directions. As a consequence, the density of states at the Fermi level is very small: 2.16 states/eV/unit cell, again in agreement with Boulet $^{17}$ calculations and with lowtemperature specific-heat measurements $\left(2.98^{2}\right)$.

The projected states on atomic orbitals have been analyzed, and the results are reported in Fig. 2. At the Fermi level, $d$-states of nickel and $s p$-states of silicon are hybridized. This may be explained by the local environment of silicon (six first neighbors). This result is compatible with a recent study of the electronic states of $\mathrm{NiSi}^{22}$

\section{B. Vibrational properties}

We have calculated the lattice dynamical properties by means of the DFPT (ESPRESSO packages ${ }^{7}$ ) with which the dynamical matrices at any $\mathbf{q}$ point can be directly computed. We have calculated interatomic force constants (IFC) by Fourier transformation of the dynamical matrices on a 2 $\times 4 \times 2$ q-mesh with a $10 \times 10 \times 10$ k-mesh and 30 Ryd cutoff energy to compute the full phonon dispersions. The vibrational density of states has been then calculated on a 30 $\times 30 \times 30$ tetrahedron grid (plotted Fig. 4). To verify the accuracy of our simulations, we have calculated directly frequencies at different $\mathbf{q}$ points $(\mathbf{X}, \mathbf{Y}$, and $\mathbf{Z})$ with a large $k$-mesh $(15 \times 15 \times 15)$ and cut-off energy (40 Ryd), without significant differences (less 5\%). Results have been reported Fig. 4 (dots).

The main experimental results are Raman spectra measured by Zhao et al. ${ }^{23}$ From a symmetry analysis, only "gerade" modes could be Raman active $\left(A_{g}\right.$ and $\left.B_{g}\right)$. We have summarized our results in Table IV and compared them to experimental data. One can notice that simulations are in very good agreement with experiments. We have analyzed the vibrational projected density of states. At high frequencies $\left(250-400 \mathrm{~cm}^{-1}\right)$, the main contribution is associated to silicon atoms, while the main contribution of nickel is at low frequencies. $^{24}$

From the vibrational density of states, we have also computed the free energy and the heat capacity (see Fig. 5):

$$
C_{v}(T)=-\left.T \frac{\partial^{2} F_{\mathrm{vib}}}{\partial T^{2}}\right|_{V}=-k_{B} \sum\left(\frac{\xi_{\mathbf{q}, \nu}}{\sinh \left(\xi_{\mathbf{q}, \nu}\right)}\right)^{2},
$$

where $\xi_{\mathbf{q}, \nu}=\hbar \omega_{q, \nu} / 2 k_{B} T$. These results allow us to calculate the Debye temperature (see Sec. III F).

\section{Elastic constants of the crystal}

As a test, we have calculated elastic constants for the two reference states. Our results are found in agreement with experimental values. ${ }^{18}$ For the silicon diamond phase, however, $C_{44}$ is found too large. It has been shown (see in particular Ref. 25) that internal atomic relaxations can strongly modify and improve elastic constants in some cases. Letting the crystal relax at each straining step yields a corrected value, $C_{44}=76 \mathrm{GPa}$, in much better agreement with experimental data $(79 \mathrm{GPa})$. It appears thus very important to take into account atomic relaxations. 
TABLE III. The calculated and experimental lattice parameters (in $\AA$ ), volume per atom (in $\AA^{3} /$ at.), formation energies $E_{f}$ per atom (in meV/at.), and magnetic moments (in $\mu_{B}$ ).

\begin{tabular}{|c|c|c|c|c|c|c|}
\hline & $a_{o}$ & $b_{o}$ & $c_{o}$ & $V$ & $E_{f}$ & $\mu_{B}$ \\
\hline Si-d (expt. $\left.{ }^{a}\right)$ & 5.43 & & & 20.09 & & \\
\hline Si-d (PAW) & 5.47 & & & 20.45 & 0 & \\
\hline Si-d (US) & 5.47 & & & 20.45 & 0 & \\
\hline $\mathrm{Ni}-\mathrm{fcc}\left(\operatorname{expt}^{\mathrm{a}}\right)$ & 3.51 & & & 10.81 & & 0.62 \\
\hline Ni-fcc (PAW) & 3.52 & & & 10.90 & 0 & 0.63 \\
\hline Ni-fcc (US) & 3.52 & & & 10.90 & 0 & 0.63 \\
\hline NiSi (expt. $\left.{ }^{b}\right)$ & 5.333 & 3.259 & 5.665 & 12.31 & $-447^{\mathrm{c}}$ & \\
\hline NiSi (expt. $\left.{ }^{d}\right)$ & 5.233 & 3.258 & 5.659 & 12.06 & & \\
\hline NiSi (expt. $\left.{ }^{e}\right)$ & 5.177 & 3.325 & 5.616 & 12.08 & & \\
\hline NiSi (PAW) & 5.179 & 3.365 & 5.613 & 12.22 & -496 & 0.00 \\
\hline NiSi (US) & 5.165 & 3.378 & 5.621 & 12.26 & -503 & 0.00 \\
\hline NiSi (theor $\left.{ }^{f}\right)$ & 5.154 & 3.343 & 5.590 & 12.04 & & 0.00 \\
\hline
\end{tabular}

\section{Reference 18. \\ ${ }^{\mathrm{b}}$ Reference 19. \\ 'Reference 20. \\ ${ }^{\mathrm{d}}$ Reference 4. \\ eReference 3. \\ ${ }^{\mathrm{f}}$ Reference 17.}

We have then adopted three approaches to calculate elastic constants for NiSi: in the first approach, we have fixed atomic positions, and in the second one, for each deformation $\delta$ applied to the system, atomic positions have been relaxed (label "relaxed" in the following, only computed with the VASP package). In the third we deduce $C_{i j}$ from slopes of the phonon dispersion curves around $\Gamma$. The results of our simulations have been summarized in Table V. In this table we have given the result of the fit of the last three deformations $\left(D_{7}, D_{8}\right.$, and $\left.D_{9}\right)$, which correspond to a combination of different elastic constants. These combinations

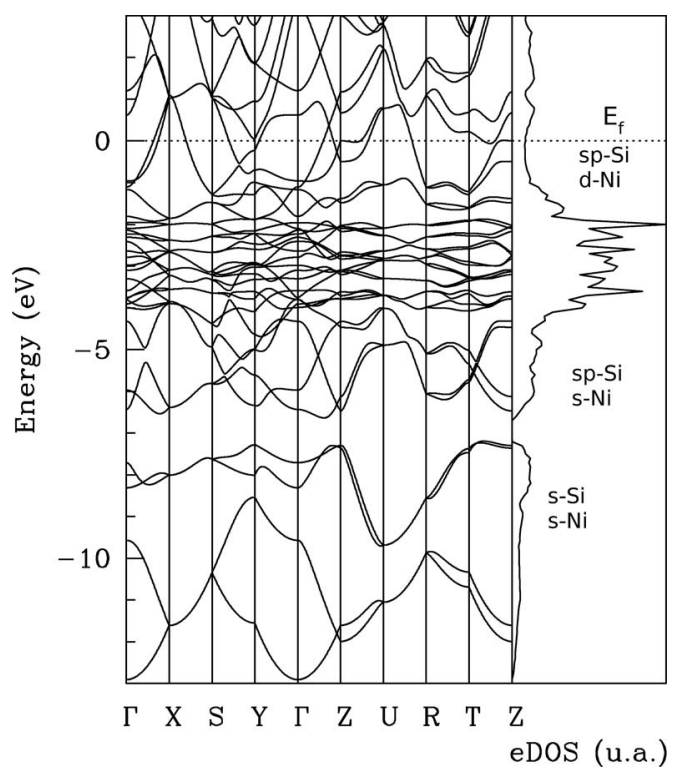

FIG. 2. Electronic band structure and density of states of NiSi. The Fermi level has been put at the zero energy. are related to criteria of mechanical stability. In the $o$-NiSi case, all are found positive.

In the first simulations, where atomic positions are not relaxed, one can notice that VASP and ESPRESSO packages produce identical values of elastic constants. The bulk modulus has been evaluated either directly from the Murnaghan equations $^{26}$ or from elastic constants calculated previously:

$$
B_{V}=\frac{1}{9}\left(C_{11}+C_{22}+C_{33}+2 C_{12}+2 C_{13}+2 C_{23}\right) .
$$

The same value is found: $168 \mathrm{GPa}$ to be compared with $175 \mathrm{GPa}$. These results are good tests of the accuracy of our simulations. In the following, we focus our analysis only on PAW simulations.

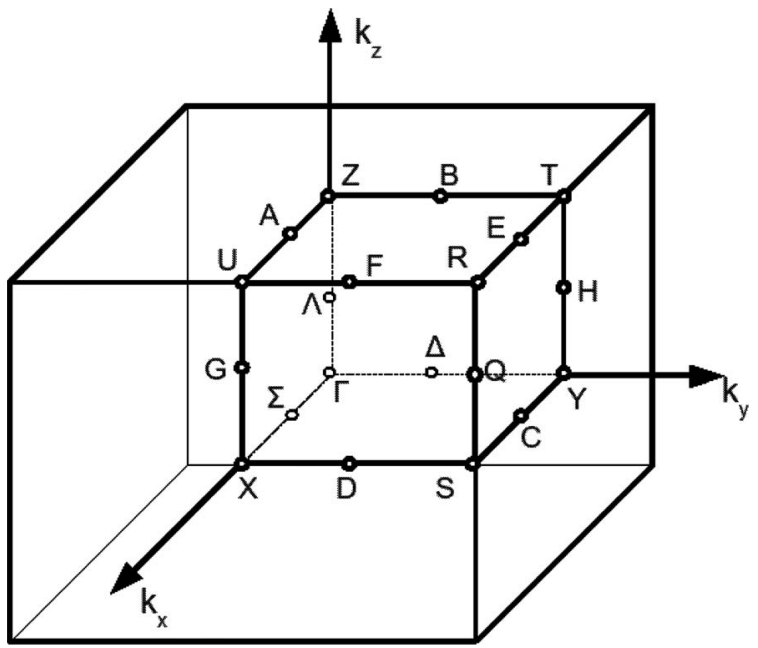

FIG. 3. Schematic representation of the high-symmetry points in the first Brillouin zone for orthorhombic systems. 


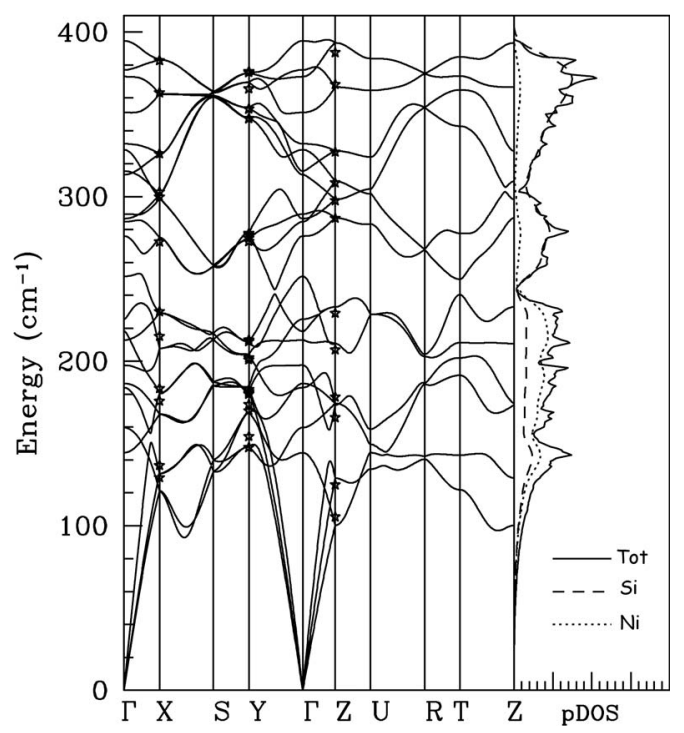

FIG. 4. Vibrational band structure and the total density of states associated (line). We have plotted the projected density of states on nickel and silicon atoms.

In a second step the influence of atomic relaxations on elastic constants has been evaluated. It is clear that, as for silicon diamond phase, elastic constants are strongly modified. The influence of relaxation ranges from $+18 \%$ to $-18 \%$. In general, we note a decrease in the elastic constants except for two elastic constants $C_{12}$ and $C_{23}$. These results are confirmed by elastic constants determined from phonon dispersion curves. In these calculations, atoms are not clamped. These results could be fruitfully compared with the relaxed simulations.

\section{Elastic properties for polycrystalline aggregates}

In the particular case of randomly oriented polycrystals, one may evaluate aggregate average elastic properties based on additional hypotheses such as isostress named as Reuss or isostrain named as Voigt states (subscripted, respectively, $V$ and $R$ in the following). For orthorhombic crystals, the shear modulus $(G)$ and the bulk modulus $(B)$ are given for each approximation by Eq. (4) and by

$$
\begin{aligned}
G_{V}= & \frac{1}{15}\left[C_{11}+C_{22}+C_{33}-\left(C_{12}+C_{13}+C_{23}\right)\right] \\
& +\frac{3}{15}\left(C_{44}+C_{55}+C_{66}\right),
\end{aligned}
$$

TABLE IV. Symmetry and frequencies $\left(\right.$ in $\mathrm{cm}^{-1}$ ) in $q=\Gamma$.

\begin{tabular}{lcccccc}
$A_{g}(R)$ & 150 & 194 & 214 & 286 & 330 & 351 \\
$A_{u}(I)$ & 0 & 163 & 214 & 271 & 285 & 374 \\
$B_{g}(R)$ & 193 & 219 & 251 & 312 & 330 & 397 \\
$B_{u}(I)$ & 0 & 0 & 182 & 290 & 316 & 377 \\
Expt. $^{\mathrm{a}}$ & 195 & 214 & 258 & 294 & 332 & 362 \\
\hline \hline
\end{tabular}

${ }^{\mathrm{a}}$ Reference 23 .
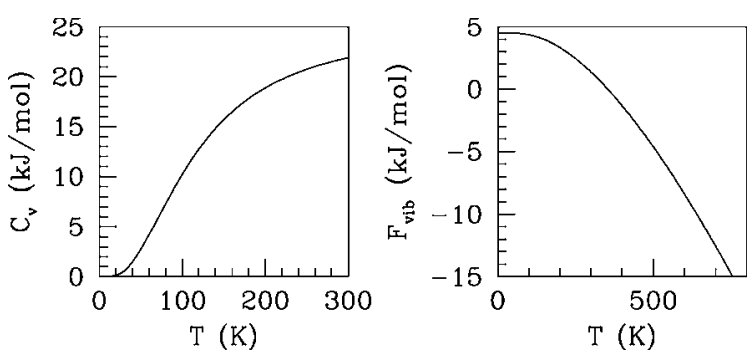

FIG. 5. Heat capacity and free energy in $\mathrm{kJ} / \mathrm{mol}$.

$$
\begin{aligned}
\frac{1}{G_{R}}= & \frac{1}{15}\left[4\left(S_{11}+S_{22}+S_{33}\right)+3\left(S_{44}+S_{55}+S_{66}\right)\right. \\
& \left.-4\left(S_{12}+S_{13}+S_{23}\right)\right], \\
\frac{1}{B_{R}} & =S_{11}+S_{22}+S_{33}+2\left(S_{12}+S_{13}+S_{23}\right),
\end{aligned}
$$

where $\mathbf{S}$ is the compliance matrix and $\mathbf{C}$ is the elastic constant matrix related by $\mathbf{S}=\mathbf{C}^{-1}$.

The calculated values are given in Table VII. Reuss and Voigt approximations produce equivalent results when atomic positions are not relaxed. On the contrary for relaxed simulations, we can see that shear modulus is smaller in Reuss approximation than in Voigt approximation.

The average of the Voigt and the Reuss bounds are considered to be the best theoretical polycrystalline elastic modulus (Hill's approximation). They are given by

$$
G_{H}=\frac{1}{2}\left(G_{V}+G_{R}\right)
$$

and

$$
B_{H}=\frac{1}{2}\left(B_{V}+B_{R}\right) .
$$

Using energy considerations, Hill showed that the Voigt and Reuss equations represent upper and lower limits of realistic polycrystalline constants and has recommended that a practical estimate of the bulk and shear moduli should be the arithmetic means of the extremes. The isotropic bulk and shear moduli for the NiSi polycrystals have been reported in Table V.

To complete the physical elastic properties we have computed the polycrystalline elastic modulus $(E)$ and the Poisson's ratio $(\nu)$ from the Hill's values using the relationships,

$$
\begin{gathered}
E=\frac{9 B_{H} G_{H}}{3 B_{H}+G_{H}}, \\
\nu=\frac{3 B_{H}-2 G_{H}}{2\left(3 B_{H}+G_{H}\right)} .
\end{gathered}
$$

Using these relations the calculated bulk modulus $B$, shear modulus $G$, Young's modulus $E$, and Poisson's ratio $\nu$ are summarized in Table VI. Elastic moduli with relaxations are smaller than without relaxations. In general the large value of shear moduli is an indication of the more pronounced directional bonding between atoms. The calculated shear moduli from relaxed and nonrelaxed single-crystal elastic constants are larger than that of most of the intermetallic compounds. 
TABLE V. Elastic constants (given in GPa), shear constants, and the bulk modulus $B_{o}$ (in GPa). For the silicon diamond phase, we report $C_{44}$ from simulations taking into account atomic relaxations (labeled as ${ }^{*}$ ).

\begin{tabular}{|c|c|c|c|c|c|c|c|c|c|c|c|}
\hline & \multicolumn{3}{|c|}{$\mathrm{Si}(\mathrm{d})$} & \multicolumn{3}{|c|}{$\mathrm{Ni}(\mathrm{fcc})$} & \multicolumn{5}{|c|}{ NiSi (ortho) } \\
\hline & Expt. $^{a}$ & PAW & US & Expt. $^{a}$ & PAW & US & & $\begin{array}{r}\text { PAW } \\
\text { Relaxed }\end{array}$ & $\Delta(\%)$ & US & DFTP \\
\hline$C_{11}$ & 166 & 154 & 152 & 250 & 274 & 271 & 283 & 272 & +4 & 285 & 275 \\
\hline$C_{22}$ & & & & & & & 248 & 222 & +10 & 244 & 205 \\
\hline$C_{33}$ & & & & & & & 281 & 229 & +18 & 278 & 269 \\
\hline$C_{44}$ & 79 & $98 / 76^{*}$ & 99 & 131 & 130 & 127 & 123 & 106 & +14 & 124 & 113 \\
\hline$C_{55}$ & & & & & & & 139 & 128 & +8 & 137 & 125 \\
\hline$C_{66}$ & & & & & & & 127 & 126 & +0 & 126 & 124 \\
\hline$C_{12}$ & 64 & 58 & 56 & 150 & 160 & 155 & 147 & 166 & -13 & 148 & \\
\hline$C_{13}$ & & & & & & & 112 & 94 & +16 & 111 & \\
\hline$C_{23}$ & & & & & & & 126 & 149 & -18 & 124 & \\
\hline$C_{11}+C_{22}-2 C_{12}$ & & & & & & & 238 & 161 & +32 & 234 & \\
\hline$C_{11}+C_{33}-2 C_{13}$ & & & & & & & 340 & 314 & +7 & 341 & \\
\hline$C_{22}+C_{33}-2 C_{23}$ & & & & & & & 278 & 152 & +45 & 274 & \\
\hline$B_{o}^{\text {Murn. }}$ & 99 & 90 & 88 & 186 & 198 & 194 & 168 & & & & \\
\hline$C^{\prime}$ & 51 & 48 & 48 & 50 & 57 & 58 & & & & & \\
\hline
\end{tabular}

${ }^{\mathrm{a}}$ Reference 18.

Poisson's ratio $\nu$ measures the stability of a crystal against shear. Its small value indicates that $o$-NiSi is relatively stable against shear.

$\mathrm{Pugh}^{27}$ proposed the ratio of bulk to shear modulus of polycrystalline phases, $B / G$ as an indication of ductile vs brittle characters. Indeed one may argue that the shear modulus $G$ represents the resistance to plastic deformation, while the bulk modulus $B$ represents the resistance to fracture. A high $B / G$ value indicates a thus tendency for ductility, while a low value indicates a tendency for brittleness. The critical value which separates ductile and brittle materials has been evaluated to be equal to 1.75 . It is interesting to notice that in nonrelaxed simulations $B / G \simeq 1.75$, the critical value, while it is found greater (2.42) with relaxation. This result suggests that $o-\mathrm{NiSi}$ is more prone to ductility.

A comparison of average elastic properties of $\mathrm{NiSi}\left(G_{H}\right.$ $=79 \mathrm{GPa}, B_{H}=170 \mathrm{GPa}, E=205 \mathrm{GPa}$, and $\left.\nu=0.30\right)$ with those of isostructural PtSi $\left(G_{H}=80 \mathrm{GPa}, B_{H}=198 \mathrm{GPa}, E\right.$ $=211 \mathrm{GPa}$, and $\nu=0.32)$ shows that these two compounds share very similar average elastic properties. As shown in the next paragraph, this similarity is lost when it comes to the anisotropy in elastic moduli.

\section{E. Quantification of the anisotropy in elastic constants}

To quantify the anisotropy of elastic properties in $o-\mathrm{NiSi}$, we have employed different criteria.

Essentially all known crystals are elastically anisotropic, and a proper description of such an anisotropic behavior has, therefore, an important implication in engineering science as well as in crystal physics. The shear anisotropic factors provide a measure of the degree of anisotropy in the bonding between atoms in different planes. The shear anisotropic factor for the $\{100\}(\{010\},\{001\})$ shear planes between the $\langle 011\rangle$ and $\langle 010\rangle\langle\langle 101\rangle,\langle 001\rangle$, and $\langle 110\rangle,\langle 010\rangle$, respectively) directions are

$$
\begin{aligned}
& A_{1}=\frac{4 C_{44}}{C_{11}+C_{33}-2 C_{13}}, \\
& A_{2}=\frac{4 C_{55}}{C_{22}+C_{33}-2 C_{23}}, \\
& A_{3}=\frac{4 C_{66}}{C_{11}+C_{22}-2 C_{12}} .
\end{aligned}
$$

TABLE VI. Isotropic value of shear and bulk moduli (in GPa) using the approximations of Voigt, Reuss, and Hill (noted $V, R$, and $H$

\begin{tabular}{|c|c|c|c|c|c|c|c|c|c|c|c|}
\hline & $G_{R}$ & $G_{V}$ & $G_{H}$ & $B_{R}$ & $B_{V}$ & $B_{H}$ & $E$ & $\nu$ & $B_{a}$ & $B_{b}$ & $B_{c}$ \\
\hline Not relaxed & 95 & 106 & 101 & 175 & 175 & 175 & 254 & 0.26 & 587 & 493 & 506 \\
\hline Relaxed & 65 & 93 & 79 & 168 & 171 & 170 & 205 & 0.30 & 486 & 957 & 352 \\
\hline PtSi $\left(\right.$ relaxed $\left.^{\mathrm{a}}\right)$ & 77 & 82 & 80 & 198 & 198 & 198 & 211 & 0.32 & 572 & 575 & 639 \\
\hline
\end{tabular}
respectively). The Young modulus (in GPa), the Poisson's ratio $\nu$, and the bulk modulus (in GPa) along crystallographic axes $a, b, c$ (see appendix of Ref. 14) are also given. We give for comparison results for PtSi (Ref. 25).

${ }^{\mathrm{a} R e f e r e n c e} 25$. 
TABLE VII. Anisotropy in shear elastic factor $\left(A_{i}\right.$ with $\left.i=1,2,3\right)$, anisotropy in directional bulk modulus $\left(A_{B_{a}} A_{B_{c}}\right)$, and anisotropy in compressibility and shear moduli $\left(A_{B}\right.$ and $A_{G}$ in $\left.\%\right)$.

\begin{tabular}{lccccccr}
\hline \hline & $A_{1}$ & $A_{2}$ & $A_{3}$ & $A_{B_{a}}$ & $A_{B_{c}}$ & $A_{B}$ & $A_{G}$ \\
\hline Not relaxed & 1.45 & 2.00 & 2.13 & 1.19 & 1.03 & 0.1 & 5.5 \\
Relaxed & 1.35 & 3.37 & 3.13 & 0.51 & 0.37 & 1.0 & 17.7 \\
\hline \hline
\end{tabular}

The shear anisotropic factors are summarized in Table VII. In the case of isotropic crystals, the factors $A_{1}, A_{2}$, and $A_{3}$ must be one, while any value smaller or greater than unity is a measure of the degree of elastic anisotropy possessed by the crystal. It is interesting to note that relaxed simulations shear anisotropic factors show a higher degree of anisotropy than nonrelaxed simulations.

Chung and Buessem ${ }^{28}$ introduced another concept of percent elastic anisotropy for noncubic systems which is a measure of elastic anisotropy possessed by the crystal under consideration. The percentage anisotropy in compressibility and shear moduli are defined, respectively, by $A_{B}=\left(B_{V}\right.$ $\left.-B_{R}\right) /\left(B_{V}+B_{R}\right)$ and $A_{G}=\left(G_{V}-G_{R}\right) /\left(G_{V}+G_{R}\right)$. A value of zero $\left(B_{R}=B_{V}\right)$ is associated to isotropic elastic constants, while a value of $100 \%$ is associated to the largest possible anisotropy. With those values of anisotropy, relaxed simulations indicate that $o$-NiSi is anisotropic $(\simeq 18 \%)$.

We have calculated the Young modulus along different directions in Table VIII. PtSi values from ${ }^{25}$ are also reported for comparison. PtSi is isostructural with NiSi. The ratio between the Young moduli along [111] and along [010] is 3.7 for $\mathrm{NiSi}$, while it is 1.5 in $\mathrm{PtSi}$. The same ratio in cubic copper, which is known to be a highly elastically anisotropic material, yields 2.9. Figure 6 is a three-dimensional (3D) representation of the anisotropy in Young's moduli for NiSi and PtSi.

The large elastic anisotropy of NiSi that we evidence here has important consequences for the mechanical properties of $\mathrm{NiSi}$ thin films. Indeed it is known that such films are strongly textured. ${ }^{29}$ Moreover the anisotropy in elastic constants together with the anisotropy in thermal expansion coefficients may yield huge grain-to-grain stress variations in polycrystal films.

Ravindran ${ }^{14}$ defined and calculated the linear bulk modulus along the three directions $a, b, c$ in orthorhombic $\mathrm{TiSi}_{2}$ system. In the same way we report them for NiSi in Table VI. If in nonrelaxed simulations no significant differences between the bulk moduli along the three directions $a, b, c$ can be observed, with relaxed results the bulk modulus along $b$ direction is much higher than in other directions.

From this directional bulk modulus, Ravindran ${ }^{14}$ also defined for orthorhombic system two new quantities, $A_{B_{a}}$ and

TABLE VIII. Young's modulus (in GPa) along different crystallographic directions, comparison of NiSi and PtSi (from Ref. 25).

\begin{tabular}{lcccc}
\hline \hline & $E_{100}$ & $E_{010}$ & $E_{001}$ & $E_{111}$ \\
\hline NiSi (relaxed) & 146 & 78 & 127 & 289 \\
PtSi (relaxed) & 201 & 150 & 200 & 226 \\
\hline \hline
\end{tabular}

$A_{B_{c}}$, defined as the ratio between $B_{a}\left(B_{c}\right)$ and $B_{b}$. A value of 1 indicates, for those quantities, elastic isotropy and any departure from one corresponds to a degree of elastic anisotropy. It is interesting again to notice that relaxed simulations indicate a much larger anisotropy.

We show that relaxed simulations indicate a pronounced elastic anisotropic system for $o-\mathrm{NiSi}$, in particular along the $b$ axis.

In conclusion, our calculations show that, at variance with isostructural PtSi, NiSi is a highly elastically anisotropic material. The ratio between the Young's moduli along [111] and [010] is 3.7, while the ratio between the bulk moduli along [001] and [010] is 0.37. It is interesting to note that the same trend is experimentally known to occur for thermal expansion ${ }^{19}$ with PtSi being much less anisotropic than NiSi.

The elastic constants we have calculated here allow for the calculation of stress and strain in a NiSi crystal under any kind of loading using linear elasticity. NiSi films are, however, polycrystalline. It is thus necessary to include a grain interaction model in order to rationalize experimental variations in interplanar spacings ${ }^{23}$ measured by $\mathrm{x}$-ray diffraction as a function of temperature. The fact that elastic constants were unknown has inhibited such studies so far.

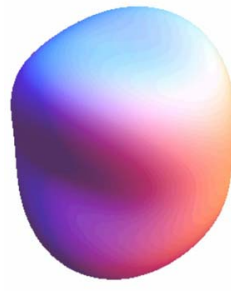

PtSi

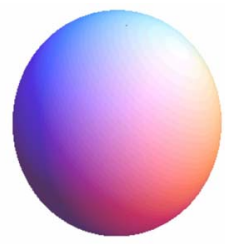

PtSi

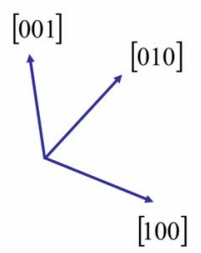

[100]

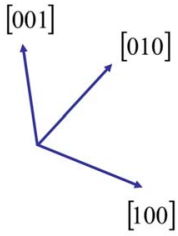

$[100]$

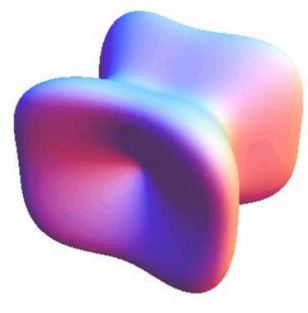

$\mathrm{NiSi}$

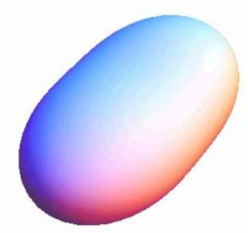

$\mathrm{NiSi}$
FIG. 6. (Color online) 3D representation of the Young modulus $E$ (top) and Bulk modulus $B$ (bottom), comparison of PtSi from Ref. 25 and NiSi. 


\section{F. Debye temperature}

We have estimated the Debye temperature $\left(\theta_{D}\right)$ from the averaged sound velocity $\left(v_{m}\right)$ using the following equation:

$$
\theta_{D}=\frac{h}{k_{B}}\left(\frac{3}{4 \pi V_{a}}\right)^{1 / 3} v_{m},
$$

where $h$ and $k_{B}$ are, respectively, Planck's and Boltzmann's constants and $V_{a}$ is the atomic volume. The average sound velocity in polycrystalline systems, $v_{m}$, are evaluated by

$$
v_{m}=\left[\frac{1}{3}\left(\frac{2}{v_{t}^{3}}+\frac{1}{v_{l}^{3}}\right)\right]^{-1 / 3},
$$

where $v_{t}$ and $v_{l}$ are the mean longitudinal and transverse sound velocities, which can be related by the shear and bulk moduli from Navier's equations:

$$
v_{l}=\left(\frac{3 B+4 G}{3 \rho}\right)^{1 / 2} \text { and } v_{t}=\left(\frac{G}{\rho}\right)^{1 / 2} .
$$

Results have been summarized in Table IX. The relaxed Debye temperature is $528 \mathrm{~K}$. In order to obtain an other estimate of the Debye temperature from vibrational properties, we have fitted the specific heat presented above with the Debye model. This fitting results in predicted values of around $480 \mathrm{~K}$. Those results are in reasonable agreement with low-temperature specific-heat measurements ${ }^{2}$ which yields a Debye temperature of $497 \mathrm{~K}$.
TABLE IX. Volumic density (in $\mathrm{kg} / \mathrm{m}^{3}$ ), longitudinal, transverse, and average sound velocities (in $\mathrm{m} / \mathrm{s}$ ), and Debye temperature (in Kelvin).

\begin{tabular}{lccccc}
\hline \hline & & & & & $\theta_{D}$ \\
& $\rho$ & $v_{l}$ & $v_{t}$ & $v_{m}$ & $(\mathrm{~K})$ \\
\hline Not relaxed & 5877 & 7259 & 4145 & 4606 & 595 \\
Relaxed & 5877 & 6844 & 3666 & 4094 & 528 \\
\hline \hline
\end{tabular}

\section{CONCLUSION}

We have presented first-principle calculations of the $o$-NiSi ground state. The structural, electronic, vibrational, and elastic properties have been calculated by means of the DFT. The optimized lattice parameters reproduce perfectly experimental data. NiSi is found to not reveal a ferromagnetic behavior and to have a small density of states at the Fermi level. The vibrational properties and in particular the heat capacity are presented. From these results and from direct calculations, we have evaluated single-crystal elastic constants. NiSi is shown to exhibit a strong elastic anisotropy; this bears important consequences for the mechanical properties of textured NiSi thin films.

\section{ACKNOWLEDGMENT}

The authors acknowledge use of the supercomputer facilities at Calmip CICT Toulouse, France. *damien.connetable@ensiacet.fr

${ }^{1}$ C. Lavoie, F. d'Heurle, C. Detavernier, and C. Cabral, Microelectron. Eng. 70, 144 (2003).

${ }^{2}$ B. Meyer, U. Gottlieb, O. Laborde, H. Yang, J. C. Lasjaunia, A. Sulpice, and R. Madar, J. Alloys Compd. 262-263, 235 (1997).

${ }^{3}$ D. F. Wilson and O. B. Cavin, Scr. Metall. Mater. 26, 85 (1992).

${ }^{4}$ C. Rivero, P. Gergaud, O. Thomas, M. Gailhanou, B. Froment, H. Jaouen, and V. Carron, Appl. Phys. Lett. 87, 041904 (2005).

${ }^{5}$ M. Fischetti and S. Laux, J. Appl. Phys. 80, 2234 (1996).

${ }^{6}$ G. Kresse and J. Hafner, Phys. Rev. B 47, 558 (1993); 49, 14251 (1994); G. Kresse and J. Furthmüller, ibid. 54, 11169 (1996); G. Kresse and J. Furthmüller, Comput. Mater. Sci. 6, 15 (1996).

${ }^{7}$ S. Baroni, A. Dal Corso, S. de Gironcoli, P. Giannozzi, C. Cavazzoni, G. Ballabio, S. Scandolo, G. Chiarotti, P. Focher, A. Pasquarello, K. Laasonen, A. Trave, R. Car, N. Marzari, and A. Kokalj, http://www.pwscf.org/.

${ }^{8}$ G. Kresse and D. Joubert, Phys. Rev. B 59, 1758 (1999).

${ }^{9}$ D. Vanderbilt, Phys. Rev. B 41, 7892 (1990).

${ }^{10}$ A. M. Rappe, K. M. Rabe, E. Kaxiras, and J. D. Joannopoulos, Phys. Rev. B 41, 1227 (1990).

${ }^{11}$ J. P. Perdew, K. Burke, and M. Ernzerhof, Phys. Rev. Lett. 77, 3865 (1996); 78, 1396 (1997).

${ }^{12}$ H. J. Monkhorst and J. D. Pack, Phys. Rev. B 13, 5188 (1976).

${ }^{13}$ R. M. Wentzcovitch, J. L. Martins, and G. D. Price, Phys. Rev.
Lett. 70, 3947 (1993)

${ }^{14}$ P. Ravindran, L. Fast, P. A. Korzhavyi, B. Johansson, J. Wills, and O. Eriksson, J. Appl. Phys. 84, 4891 (1998).

${ }^{15}$ S. Baroni, S. de Gironcoli, A. Dal Corso, and P. Giannozzi, Rev. Mod. Phys. 73, 515 (2001).

${ }^{16}$ A. G. Every, Phys. Rev. B 22, 1746 (1980).

${ }^{17}$ R. M. Boulet, A. E. Dunsworth, J. P. Jan, and H. L. Skriver, J. Phys. F: Met. Phys. 10, 2197 (1980).

${ }^{18}$ C. Kittel, Introduction to Solid State Physics (Wiley, New York, 1996).

${ }^{19}$ C. Detavernier, C. Lavoie, and F. M. d'Heurle, J. Appl. Phys. 93, 2510 (2003).

${ }^{20}$ W. Oelson and H. Samson-Himmelstjerna, Mitt. Kaiser-Wilhelm Inst. Eisenforch. Düsseldorf 18, 131 (1936).

${ }^{21}$ T. Tokunaga, K. Nishio, H. Ohtani, and M. Hasebe, CALPHAD: Comput. Coupling Phase Diagrams Thermochem. 27, 161 (2003).

${ }^{22}$ N. Kawasaki, N. Sugiyama, Y. Otsuka, H. Hashimoto, M. Tsujimoto, H. Kurata, and S. Isoda, Ultramicroscopy 108, 399 (2008).

${ }^{23}$ F. F. Zhao, S. Y. Chen, Z. X. Shen, X. S. Gao, J. Z. Zheng, A. K. See, and L. H. Chan, J. Vac. Sci. Technol. B 21, 862 (2003).

${ }^{24}$ S. K. Donthu, S. Tripathy, D. Z. Chi, and S. J. Chua, J. Raman Spectrosc. 35, 536 (2004).

${ }^{25}$ O. Beckstein, J. E. Klepeis, G. L. W. Hart, and O. Pankratov, 
Phys. Rev. B 63, 134112 (2001).

${ }^{26}$ F. D. Murnaghan, Proc. Natl. Acad. Sci. U.S.A. 30, 244 (1944).

${ }^{27}$ S. F. Pugh, Philos. Mag. 45, 823 (1954).

${ }^{28}$ D. H. Chung and W. R. Buessem, in Anisotropy in Single Crystal Refractory Compound, edited by F. W. Vahldiek and S. A. Mer- sol (Plenum, New York, 1968), Vol. 2, p. 217.

${ }^{29}$ C. Detavernier, A. S. Özcan, J. Jordan-Sweet, E. A. Stach, J. Tersoff, F. M. Ross, and C. Lavoie, Nature (London) 426, 641 (2003). 